\title{
Study of layers' structure defects of 3D objects obtained by selective laser melting
}

\author{
Vladimir D. Gurin ${ }^{a}$, Tatiana Tyurbeeva, Pavel Yu. Peretyagin, Dmitry V. Kotoban \\ AND ANNA A. OKUNKOVA
}

Moscow State University of Technology "STANKIN", 1 Vadkovsky per., Moscow GSP-4, 127994, Russian Federation

Received 23 November 2016, Accepted 12 December 2016

\begin{abstract}
Current work was aimed to study of layers' structure defects of 3D-objects obtained by selective laser melting with the modulated laser beam mode. Traditionally Gaussian laser beam mode uses for the processing in selective laser melting. On the developed experimental setup, the alternative laser beam modes as Flat-top and Inverse Gaussian were obtained. With the carried out experiments 3D-objects contained 10 layers were produced from CoCrMo powder with the diameter of granules less than $20 \mu \mathrm{m}$. The layers' structure defects as penetration of the substrate material into the body of produced objects were studied by optical and scanning electronic microscopy.
\end{abstract}

Key words: Selective laser melting / 3D-object / laser beam mode / laser beam power density distribution / CoCrMo powder / structure defects / SEM

\section{Introduction}

Today the method of selective laser melting (SLM) is quite widespread. The principle of the method is in layer-by-layer growing of solids from mostly metallic or plastic particles to the 3D-object by laser melting of the particles in each layer according to developed program. The advantages of the method include:

- Relevant simplicity of work preparation process;

- Possibility to obtain the object with quite complex $3 \mathrm{D}$-geometry, when it is not possible or hardly possible to do it by traditional machining methods;

- Absence or significant reduction of post-operation processes;

- Possibility to obtain the final geometry of the object with only one operation step;

- Absence of the cutting tool; which can give a deviation of the geometry with its wear [1-3].

One of the main disadvantage of the process is also its costs. Meanwhile nowadays many of the manufactures reduced the costs of the powders by optimization of its production. SLM already took his niche the field of the individual application as personal medical implants, experimental and industrial parts of important mechanisms in aviation, automotive, machine-building and nuclear industries $[4,5]$. The possible defects of microstructure for CoCrMo alloys is described in the works [6-8].

\footnotetext{
a Corresponding author: lecast@stankin.ru
}

Up to now, the question of productivity and efficiency of the method is actual. Every time application of the method asks proved substantiation because of its significant part of final costs of manufacture of product. When the problem of productivity and efficiency of SLM-processing will be solved, the method will be introduced for its further perspectives and possible applications $[9,10]$.

There are several opportunities to enlarge the efficiency of SLM-processing on the way of the energy transformation:

- Direct improvement of laser system power: actually it gives the opposite effect, it reduces dramatically quality of single tracks and a final 3D-object;

- Improvement of optical elements of a machine on the laser beam way (in fact, there are no visible results);

- Improvement the absorption qualities of the powder by improvement of smoothness of granules' surface (in fact, there are no significant results).

Plenty of the scientific authors proved nowadays theoretical and practical possibility of modulation of the laser beam from laser beam mode TEM00 to alternative ones for technical and technological uses [11-18].

The current research aim of the study it is pointed to the problem of reducing of the influence of the described negative effects on the quality of the final 3D-object, obtained by SLM, and to the search of the possible improvement the processing efficiency. The research is based on 

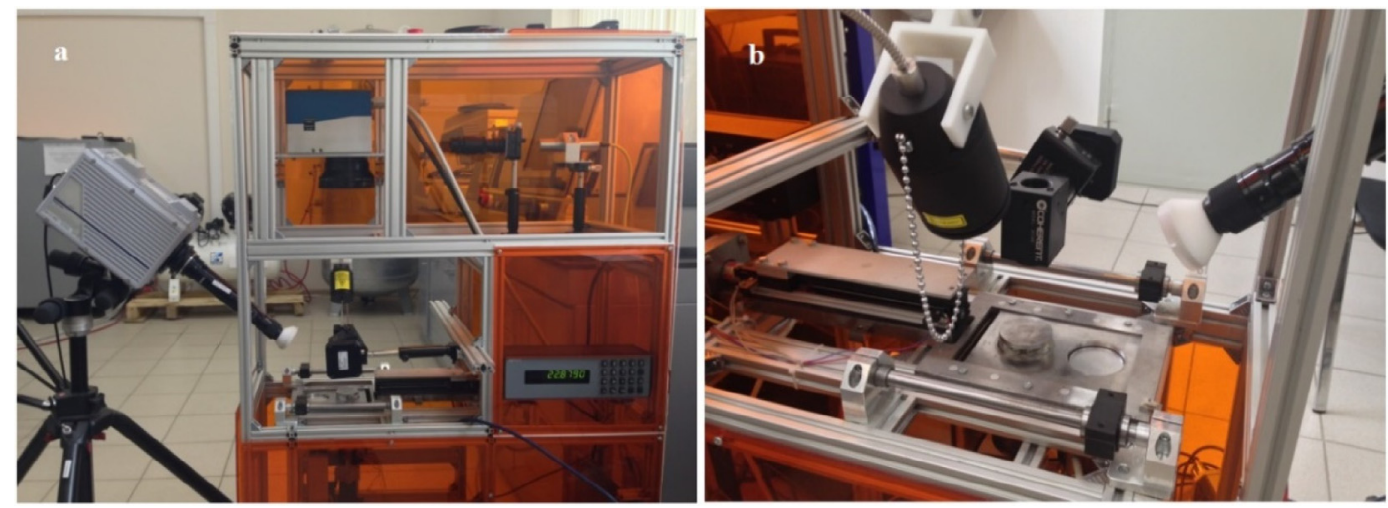

Fig. 1. Developed experimental setup with installed laser beam modulation and optical diagnostic systems: (a) general view, (b) working area.

the obtained experimental data and including the modeling of 3D technological fields in area out of the technological limits of the equipment.

\section{Experimental setup}

The experimental setup was developed for the aims of the study (Fig. 1) [19]. The setup was supplied by relevant optical and diagnostic systems for laser beam modulation and observation of the working zone.

The commercially available powder of cobalt-chrome alloy CoCrMo was used as a material for the current research work [20]. Chemical composition of the powder is: $\mathrm{Co}-60-65 \%$; Cr $-26-30 \%$; Mo $-5-7 \%$; Si $->1 \%$; Mn $>1 \%$; $\mathrm{Fe}->0.75 \%$; $\mathrm{C}->0.16 \%$; $\mathrm{Ni}->0.1 \%$. This powder was produced by means of gas atomization and has spherical particle morphology, which is more preferable for SLM uses. The powder provides a spherical morphology that was identified by SEM analysis (Tescan Vega 3 LMH, Czech Republic) and granulomorphometric investigation (OCCHIO 500 NANO, Belgium) (Fig. 2) [21]. All experimental works produced without protected atmosphere because of there were no possibility to put all monitoring and optical equipment in the camera of the setup. Meanwhile the practice said CoCrMo powder has the lowest reaction with atmosphere in comparison with other powders (Ti6Al4V alloy, maraging steel etc.).

Powder material layer nominal thickness in this study has been $30 \div 40 \mu \mathrm{m}$ and controlled by optical microscope Olympus BX51M (Japan). The main SLM-parameters and their functional dependences, identified during current study, are presented in Figure 3.

The main laser beam power density distributions (modes) as Gaussian, Flat-top and Inverse Gaussian were obtained on the experimental setup by described method $[19,20]$ and were controlled optically by CCDcamera LaserCam HRTM.

For calculation of the distance between different laser beam distributions the next formula has been used:

$$
R=\frac{8 \times \lambda \times f^{\prime 2}}{\pi \times D^{2}}
$$

where $\lambda$ - laser wave length, $f^{\prime}$ - focus distance $F$ - theta lens, $D$ - laser beam diameter after collimator.

For each of the laser beam distributions the effective diameter was measured with the purpose to calculate effective area of the laser beam spot on the surface of the powder.

Based on the results obtained in the previous work [22], the revealed technological gaps for each of the developed laser beam mode were investigated more careful with the object to discover a possibility to obtain 3Dobjects by SLM (Tab. 1). Each of the object contained 10 layers. For the SLM samples, production double hatching strategy of laser beam movement in each layer was used.

\section{Experimental results}

During the experiments, the several samples for each of the technological gap were produced. Sectioning of the samples was performed at ATM sample preparing equipment (Germany), including a comprising cutoff machine, a hydraulic press and a polishing machine. Olymlpus BX51M optical microscope and SEM Tescan Vega 3 LMH have used to study microstructure of the samples and its chemical composition. Before optical microscopy all samples were etched chemically by solution of chemically pure $\mathrm{HCl}(50 \mathrm{ml})+\mathrm{FeCl}(10 \mathrm{~g})+5$ drops of HNO3.

Figure 4 demonstrates the most typical structures, which were obtained for each of the technological gaps.

The microstructures obtained on the SLM-parameters for first technological gaps (Figs. 4a, 4c, 4e) with the scanning speed less than $30 \mathrm{~mm} / \mathrm{s}$ (Tab. 1) has visible irregularity in formation of 3D-objects. For the objects obtained in the first technological gap for Gaussian laser beam mode, the structures has more regular character, but still its formation has defects, especially in the part of first 5-6 layers of the object, where liquid metal alloys of the powder and the substrate mixed turbulently.

For the objects obtained with the parameters for Flattop and Inverse Gaussian laser beam mode (Figs. 4c, 4e) the absence of metallurgical contact between the layers in few place was observed. It shows that first technological 
V.D. Gurin et al.: Mechanics \& Industry 17, 718 (2016)

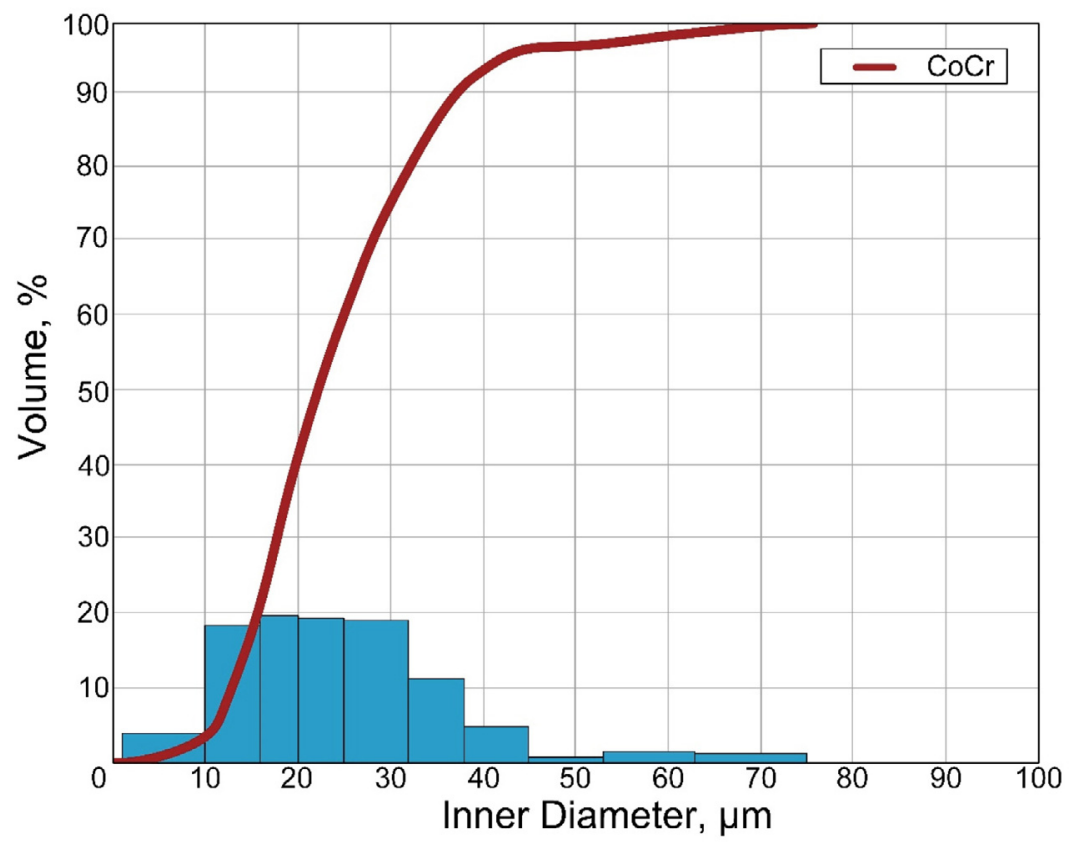

Fig. 2. Granulometric analysis of CoCrMo powder.

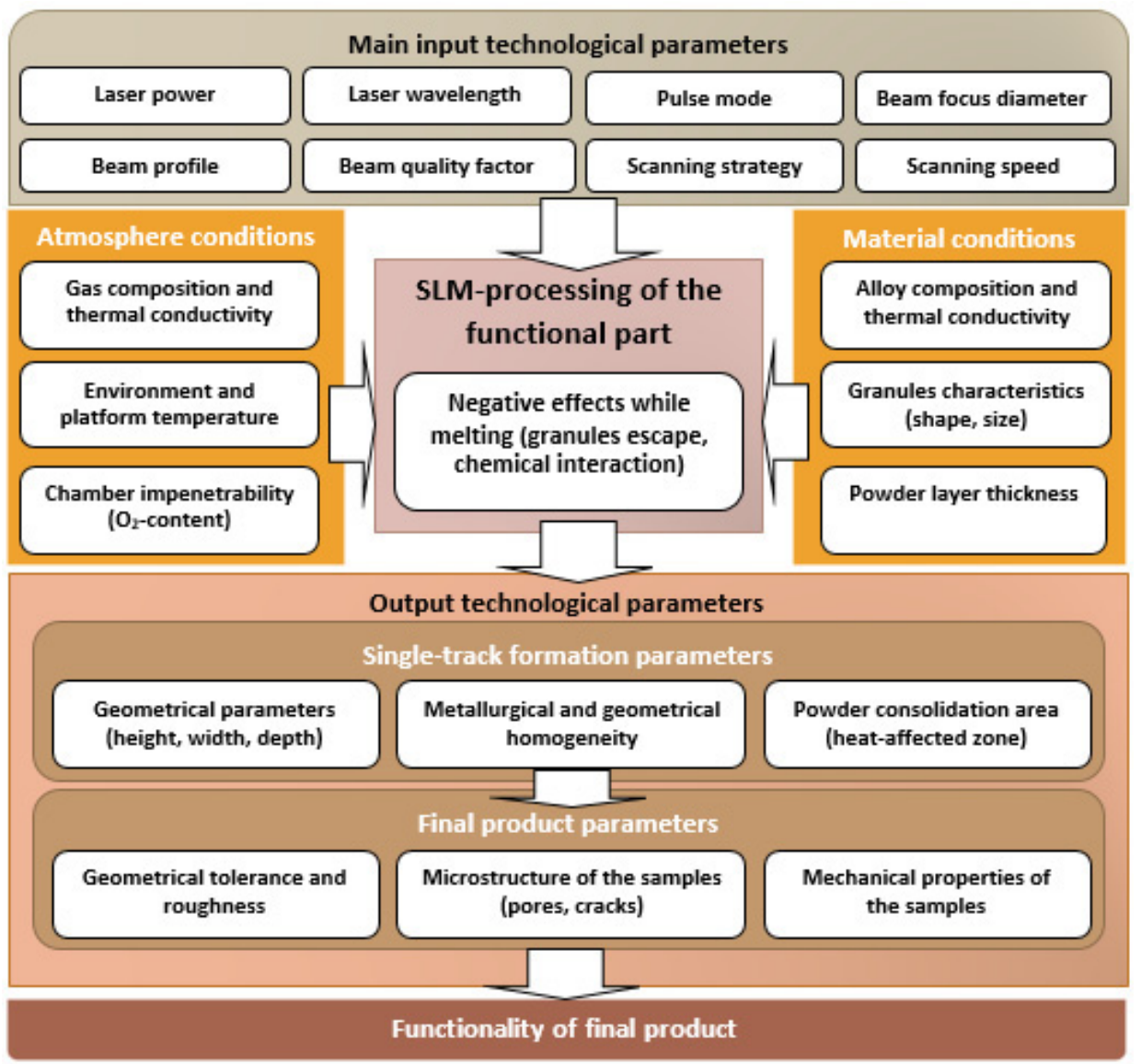

Fig. 3. Functionality of input and output parameters of selective laser melting process. 
V.D. Gurin et al.: Mechanics \& Industry 17, 718 (2016)

Table 1. Results of experimental work for single-track formation and technological gaps for SLM-processing of CoCrMo powder with different laser beam energy distribution.

\begin{tabular}{|c|c|c|c|c|c|c|c|c|c|c|c|}
\hline \multicolumn{12}{|c|}{ Gaussian laser beam distribution } \\
\hline \multicolumn{12}{|c|}{ Scanning speed $(\mathrm{V}), \mathrm{mm} / \mathrm{s}$} \\
\hline & & 5 & 10 & 20 & 30 & 40 & 50 & 60 & 70 & 80 & 100 \\
\hline & 10 & 0 & 0 & 0 & 0 & 0 & 0 & 0 & 0 & 0 & 0 \\
\hline \multirow{6}{*}{$\begin{array}{l}\hat{\theta} \\
\dot{0} \\
\tilde{0} \\
0 \\
\tilde{\Xi} \\
\tilde{\Xi} \\
0 \\
0\end{array}$} & 30 & + & + & + & - & - & - & 0 & 0 & 0 & 0 \\
\hline & 50 & + & + & + & - & - & - & - & - & - & - \\
\hline & 70 & + & + & + & + & - & - & - & - & - & - \\
\hline & 100 & ++ & ++ & - & + & - & - & - & - & - & - \\
\hline & 130 & ++ & ++ & ++ & - & - & + & + & + & - & - \\
\hline & 150 & ++ & ++ & + & + & + & + & + & + & + & - \\
\hline \multirow{2}{*}{ 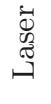 } & 170 & ++ & ++ & + & - & - & + & + & + & + & + \\
\hline & 200 & ++ & ++ & ++ & ++ & + & ++ & - & + & - & + \\
\hline \multicolumn{12}{|c|}{ Flat-top laser beam distribution } \\
\hline \multicolumn{12}{|c|}{ Scanning speed $(\mathrm{V}), \mathrm{mm} / \mathrm{s}$} \\
\hline \multirow{10}{*}{ 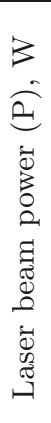 } & & 5 & 10 & 20 & 30 & 40 & 50 & 60 & 70 & 80 & 100 \\
\hline & 10 & 0 & ++ & 0 & 0 & 0 & 0 & 0 & 0 & 0 & 0 \\
\hline & 30 & + & - & - & 0 & 0 & 0 & 0 & 0 & 0 & 0 \\
\hline & 50 & - & + & - & - & - & - & - & - & - & - \\
\hline & 70 & + & + & + & - & - & - & - & - & - & 0 \\
\hline & 100 & ++ & ++ & - & - & - & - & + & + & + & + \\
\hline & 130 & ++ & ++ & ++ & + & + & + & - & + & + & 0 \\
\hline & 150 & ++ & ++ & ++ & + & - & + & + & + & - & - \\
\hline & 170 & ++ & ++ & + & + & + & + & + & + & + & 0 \\
\hline & 200 & ++ & ++ & ++ & ++ & + & + & + & + & - & + \\
\hline \multicolumn{12}{|c|}{ Inverse Gaussian laser beam distribution } \\
\hline \multicolumn{12}{|c|}{ Scanning speed $(\mathrm{V}), \mathrm{mm} / \mathrm{s}$} \\
\hline \multirow{10}{*}{ 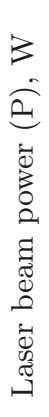 } & & 5 & 10 & 20 & 30 & 40 & 50 & 60 & 70 & 80 & 100 \\
\hline & 10 & 0 & 0 & 0 & 0 & 0 & 0 & 0 & 0 & 0 & 0 \\
\hline & 30 & 0 & 0 & 0 & 0 & 0 & 0 & 0 & 0 & 0 & 0 \\
\hline & 50 & - & - & - & - & - & 0 & 0 & 0 & 0 & 0 \\
\hline & 70 & + & + & - & - & - & - & - & - & - & - \\
\hline & 100 & + & + & + & - & + & - & - & - & - & - \\
\hline & 130 & ++ & + & + & + & - & - & - & - & 0 & - \\
\hline & 150 & ++ & ++ & ++ & - & + & - & + & + & + & - \\
\hline & 170 & ++ & ++ & ++ & + & + & + & + & + & + & + \\
\hline & 200 & ++ & ++ & - & ++ & + & + & + & + & + & + \\
\hline
\end{tabular}

Where $(0)$ is a full absence of metallurgical contact; $(+)$ is a stable track formation; $(++)$ is too deep relative penetration into the substrate; $(-)$ is non-stable metallurgical contact with subtract.

gap for the mentioned laser beam power density distribution is not preferable for growing solids by SLM. The reason of the formation of this kind of non-stable structures can be the effect of the overheating and multiply remelting of the molten pool in each layer.

Meanwhile better picture for the obtained structures of the samples was obtained for the Flat-top and Inverse Gaussian laser beam mode in the second technological gap (scanning speed is more than $40 \mathrm{~mm} / \mathrm{s}$, Tab. 1) on the Figures 4d, 4f. The structures has uniform character. The main difference between the pictures is in the depth of penetration formed molten pools in the previous layer and the thickness of the remelted and layers of substrate material and powder material. In the case of second technological gap for Inverse Gaussian laser beam mode the thickness of these layers can be hardly detected and measured as less than $50 \mu \mathrm{m}$ (Fig. 4f).

This kind of formation is shown for the images Figures $4 \mathrm{a}-4 \mathrm{~d}$, so it can be fully detected for Gaussian and Flat-top laser beam mode.

Meanwhile, if for Gaussian laser beam mode the penetration of the substrate material is measured as 150$200 \mu \mathrm{m}$ (Figs. 4a, 4b), for Flat-top laser beam mode is measured 80-100 $\mu \mathrm{m}$ (Figs. 4c, 4d). For the case of Inverse Gaussian Beam the mixed layers has thickness about 50- 


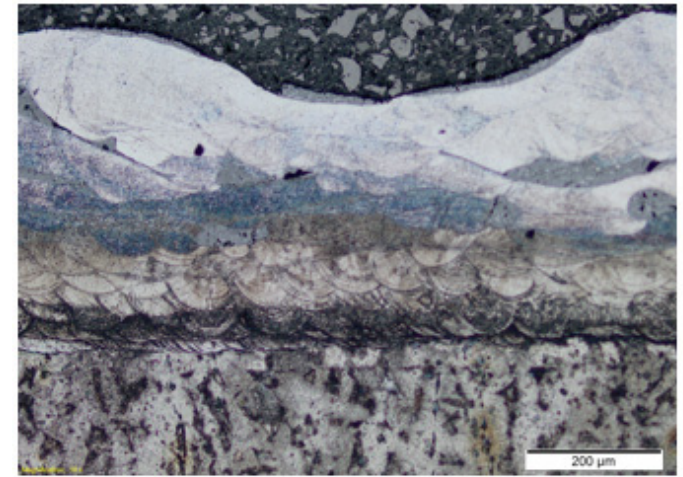

(a)

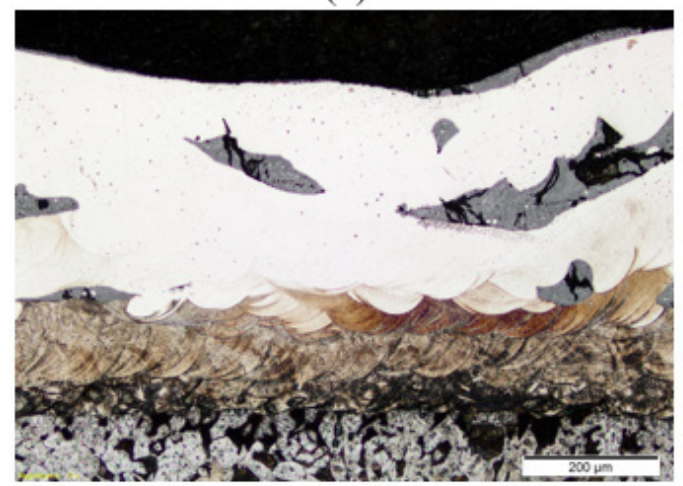

(c)

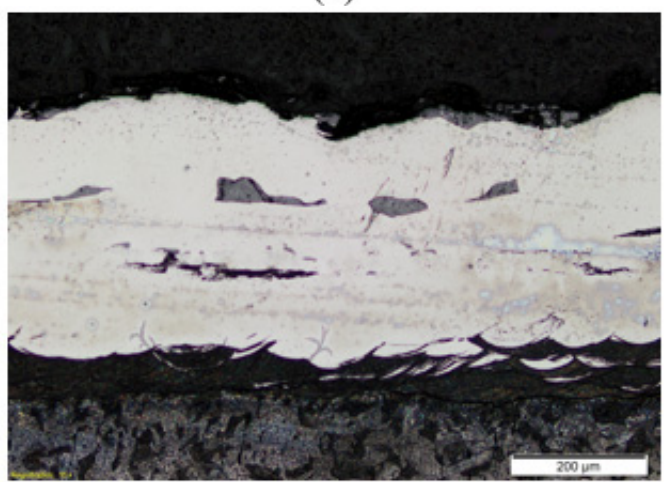

(e)

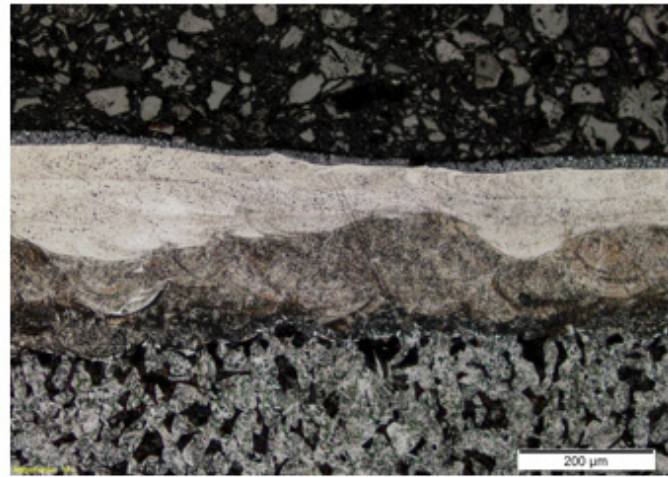

(b)

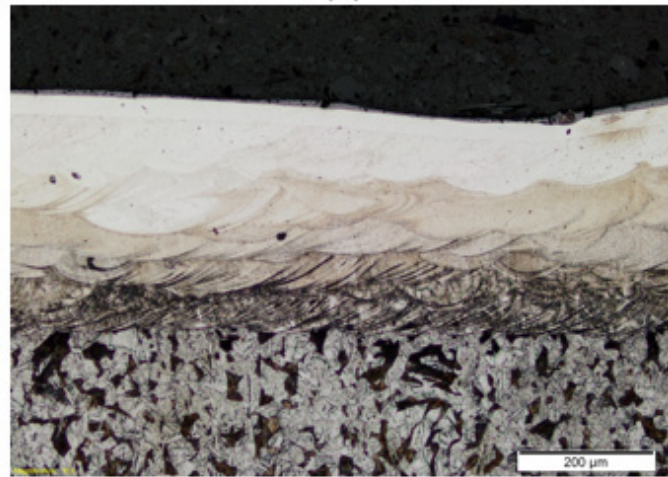

(d)

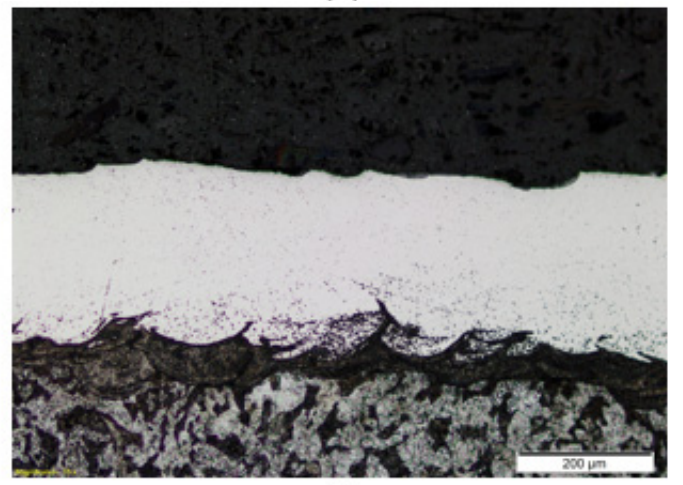

(f)

Fig. 4. Images of the optical microscopy of the samples for each of the technological gap with the following SLM-parameters: (a) for Gaussian laser beam mode $\mathrm{P}-50 \mathrm{~W}, \mathrm{~V}-20 \mathrm{~mm} / \mathrm{s}$; (b) for Gaussian laser beam mode $\mathrm{P}-170 \mathrm{~W}, \mathrm{~V}-50 \mathrm{~mm} / \mathrm{s}$; (c) for Flat-top laser beam mode P - $70 \mathrm{~W}, \mathrm{~V}-20 \mathrm{~mm} / \mathrm{s}$; (d) for Flat-top laser beam mode P - $170 \mathrm{~W}, \mathrm{~V}-60 \mathrm{~mm} / \mathrm{s}$; (e) for Inverse Gaussian laser beam mode P $-70 \mathrm{~W}, \mathrm{~V}-10 \mathrm{~mm} / \mathrm{s}$; (f) for Inverse Gaussian laser beam mode P $-170 \mathrm{~W}, \mathrm{~V}-70 \mathrm{~mm} / \mathrm{s}$.

$70 \mu \mathrm{m}$ (Figs. 4e, 4f) as it was mentioned above and it is significantly less than in the previous examples.

It can be evidence of the relative depth of the molten pool, which reduces with the changing of the mode from Gaussian to Inverse Gaussian. The depth depends on the laser beam power density distribution, which shown in $[21,22]$.

In that case, more preferable laser beam power density distribution will be the distribution, which gave a possibility to obtain a necessaire metallurgical contact between a substrate and an object on the appropriate depth (according to the technological function of the end product) up to the moment of the end of its technological us- age. Then Flat-top or Inverse Gaussian laser beam mode can be recommended for different technological purposes. For example, for thin structures from valuable materials and their easy remove from a substrate, Inverse Gaussian laser beam mode can be used. Inverse Gaussian laser beam mode can provide a metallurgical contact to finish SLM-processing, it will not give too deep penetration of the chemical composition of the substrate material into an object and the object can be easily removed from the substrate. In the case of Flat-top laser beam mode, the distribution can be recommended to produce relatively heavy objects on the substrate from the same material like deposition of the coating-like objects or growing-up 


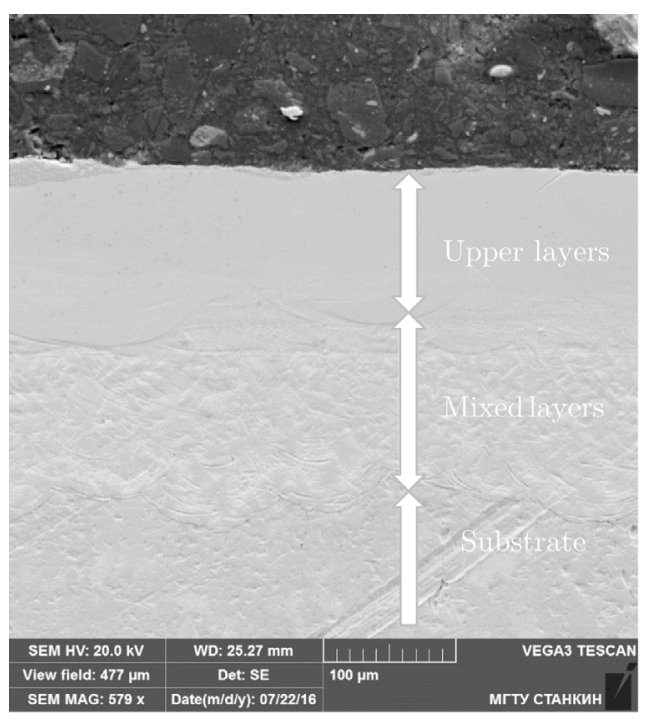

(a)

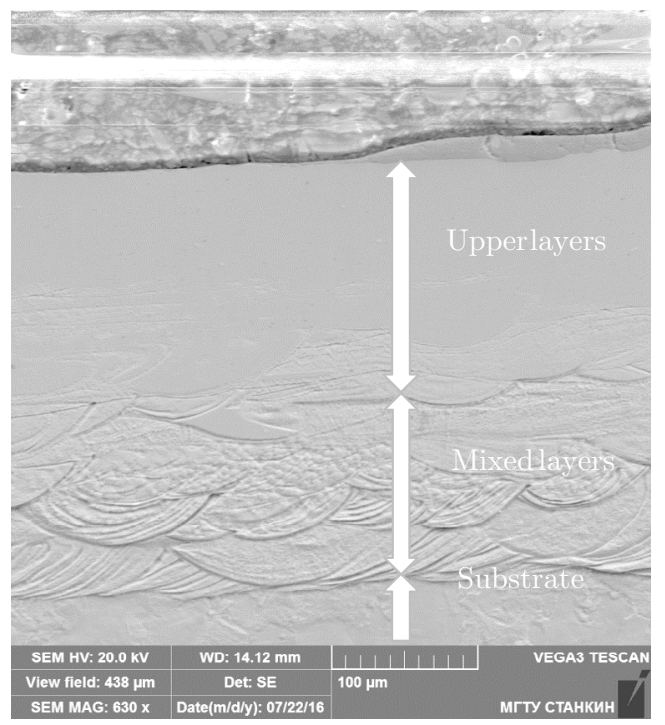

(b)

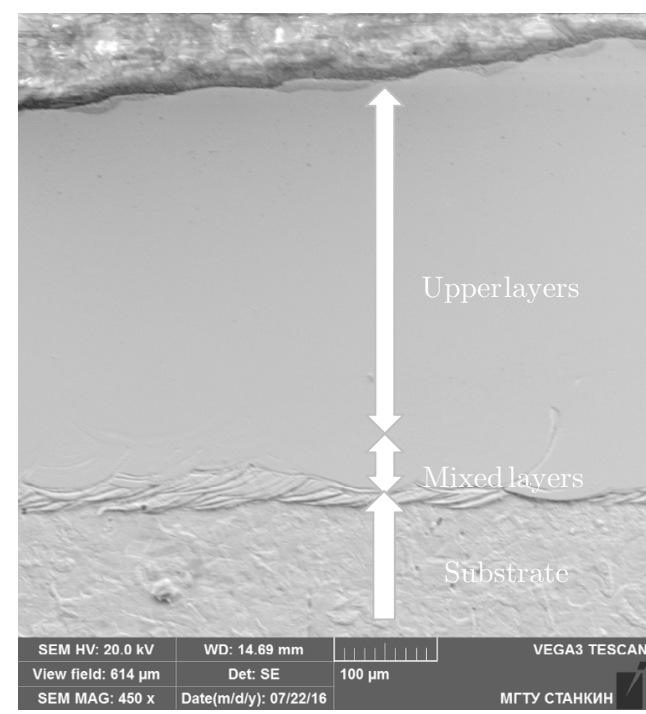

(c)

Fig. 5. SEM-images of the cross-section of the obtained samples for Gaussian (a), Flat-top (b), Inverse Gaussian (c) laser beam mode in the second technological gap, where: (a) is with $\mathrm{P}-150 \mathrm{~W}, \mathrm{~V}-80 \mathrm{~mm} / \mathrm{s}$; (b) is $\mathrm{P}-150 \mathrm{~W}, \mathrm{~V}-60 \mathrm{~mm} / \mathrm{s}$; (c) is $\mathrm{P}-$ $170 \mathrm{~W}, \mathrm{~V}-60 \mathrm{~mm} / \mathrm{s}$.

solid elements on the surface of a just-produced part for construction reasons or 3D-repairing of a worn part of an object surface.

SEM images (Fig. 5) showed even more detailed the influence of the mixed layers on the formation of the obtained structures. The picture of first 5-6 layers of the sample obtained for Gaussian laser beam mode has definitely turbulent character after multiply remelting of solid solution of materials of the substrate and the powder, then upper layers of the samples has smooth character (Fig. 5a). For Flat-top laser beam mode the thickness of mixed layers is reduced, the observed character of the structure showed significantly the picture of the layer formation: molten pool after molten pool for each layer, than the upper layer has smooth character (Fig. 5b). The usage Inverse Gaussian laser beam mode for production of the samples by SLM gives promising results, because it shows the significant reduction of the mixed layers thickness (Fig. 5c). It can be consequence of more uniform laser beam power distribution [25], which should equal distributed and absorbed by powder layer in the spot. In fact, knife-like melting and formation of the molten pool by Gaussian laser beam mode is preferable in such kind of technological operation as laser cutting (especially for thin sheets of material). For thin sheets, conical defects of sheet billet borders are mostly acceptable, when it can be not desirable for laser cutting of thick sheets, laser graving, laser micro dicing. For laser cladding and selective 


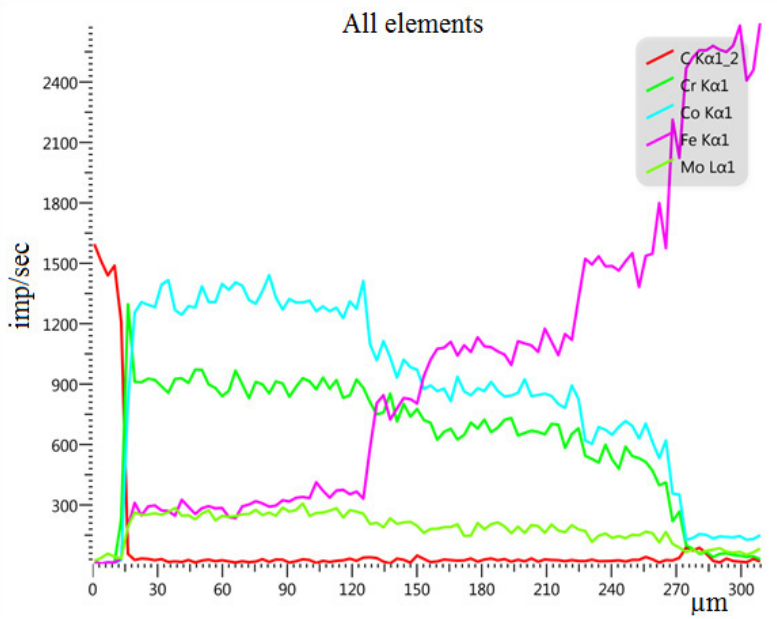

(a)

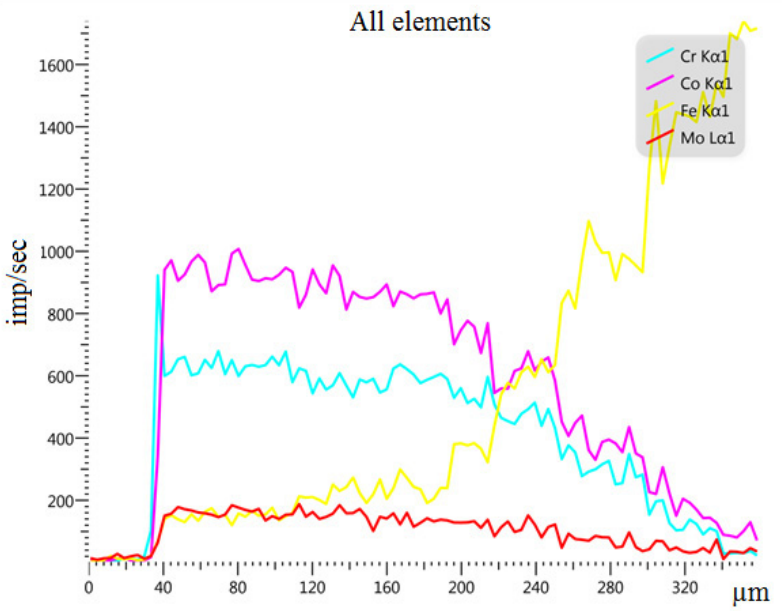

(b)

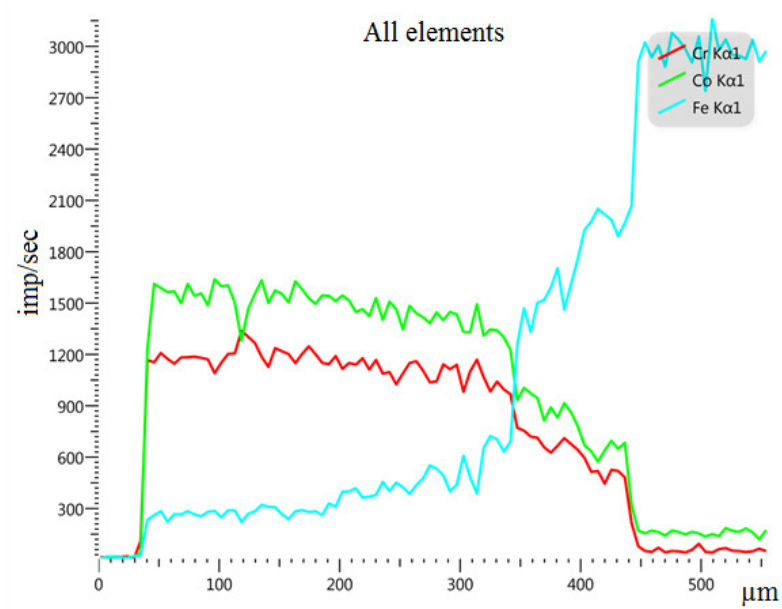

(c)

Fig. 6. Chemical analysis of the cross-section of the obtained samples for Gaussian (a), Flat-top (b), Inverse Gaussian (c) laser beam mode in the second technological gap, where: (a) is with $\mathrm{P}-150 \mathrm{~W}, \mathrm{~V}-80 \mathrm{~mm} / \mathrm{s}$; (b) is $\mathrm{P}-150 \mathrm{~W}, \mathrm{~V}-60 \mathrm{~mm} / \mathrm{s}$; (c) is $\mathrm{P}-170 \mathrm{~W}, \mathrm{~V}-60 \mathrm{~mm} / \mathrm{s}$.

laser melting, knife-like melting of the molten pool gives overheating in the center of the molten pool. In that case, the periphery of the laser beam spot on the powder surface sometimes has not enough energy to start melting process, but it involves powder granules from periphery of the molten pool by an active stream of liquid metal into the molten pool by its turbulent flows. It can be related to the convectional flows and the Gibbs-Marangoni effect. In that case, non-melted granules on the surface of the molten pool can leave it on the very high speed (shotlike) because of thermal shock in the center of the molten pool and high gradient of the temperature in the molten pool between its center and periphery. The overheating in the center of the molten pool gives also the effect of active metal evaporation which registered by optical monitoring as a bright plasma cloud above the molten pool. The cloud condensates as metallic drops around the working zone on the way of laser beam pass. The usage of Flat-top and In- verse Gaussian laser beam mode reduces the described negative effects as it was proved by first experiments on the stage of single track formation [19-24]. As it can be seen in Figure 5, alternative laser beam mode has influenced also on the thickness of mixed layer of 3D-objects. With the extension of the effective diameter of laser beam spot on the powder surface, the depth of each molten pool reduces, and the negative effects, which provoke the involvement of the material from the previous layers and substrate into the upper levels by reducing the effect of deep multiply remelting of the molten pool accompanied the process, reduce also.

Chemical analysis of samples' cross-sections for each of the laser beam mode received by SEM gives detailed data about penetration of substrate material into the obtained objects (Fig. 6). As it can be seen on the images the depth of penetration of the iron (main element of substrate material, steel 20 GOST $1050-88$ or its US 


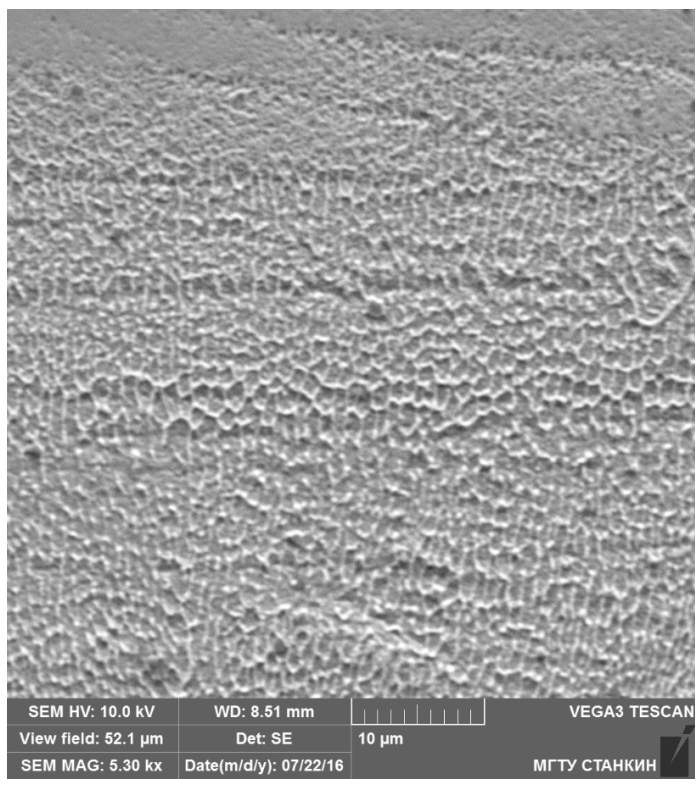

(a)

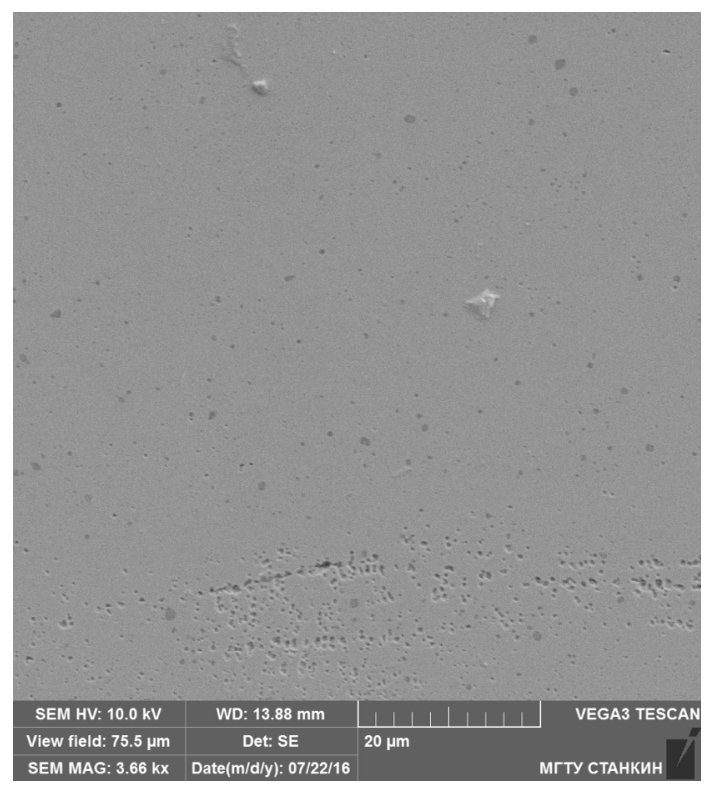

(b)

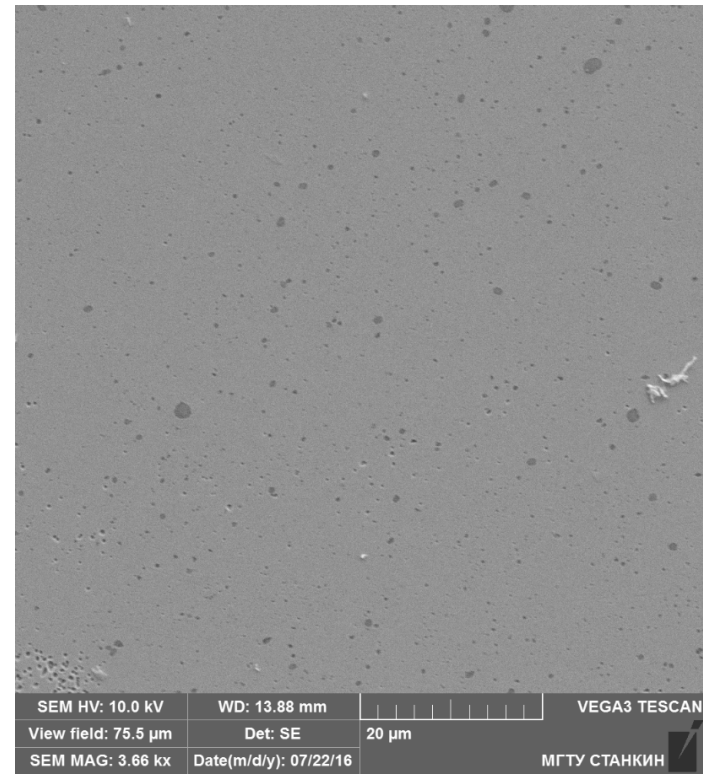

(c)

Fig. 7. SEM-images of the objects' micro defects: (a) is for Gaussian laser beam mode, where $\mathrm{P}-150 \mathrm{~W}, \mathrm{~V}-80 \mathrm{~mm} / \mathrm{s}$, (b) is for Flat-top laser beam mode, where $\mathrm{P}-150 \mathrm{~W}, \mathrm{~V}-60 \mathrm{~mm} / \mathrm{s},(\mathrm{c})$ is for Inverse Gaussian laser beam mode, where $\mathrm{P}-170 \mathrm{~W}$, $\mathrm{V}-60 \mathrm{~mm} / \mathrm{s}$.

analogue AISI C1020) reduces with change of laser beam mode (with extension of effective diameter of laser beam spot on the powder surface) and reduction of molten pool depth.

For the samples produced by Gaussian laser beam mode the measured depth of penetration of the iron is $150 \mu \mathrm{m}$ (and the thickness $120 \mu \mathrm{m}$ for upper layers), for Flat-top laser beam mode the measured depth of penetration is $120 \mu \mathrm{m}$ (and the thickness $160 \mu \mathrm{m}$ for upper layers) and for Inverse Gaussian laser beam mode the measured depth of penetration is $100 \mu \mathrm{m}$ (and the thickness $300 \mu \mathrm{m}$ for upper layers). These measures confirms the data received by optical microscopy (Fig. 4) and SEM (Fig. 5), definitely there is correlation between material reaction during etching in the mixed layers (Fig. 4), obtained structure of the mixed layers (Fig. 5) and the depth of iron penetration into the samples (Fig. 6).

As well on the graphs of Figure 6, there is a different character of the penetration for each of the laser beam mode. The level of the iron in the sample for Gaussian 
laser beam mode (Fig. 6a) increases dramatically in the range between $120-150 \mu \mathrm{m}$ of the depth from the upper surface of the sample, than the level of the iron increases gradually. In the case of Flat-top laser beam mode the level of the iron is growing gradually from $160 \mu \mathrm{m}$ of the depth from the upper surface of the sample (Fig. 6b). For Inverse Gaussian laser beam mode the level of the iron in the sample grows sharply from the depth of $300 \mu \mathrm{m}$ from the upper surface of the sample (Fig. 6c).

For technological aspects of the SLM-processing the preferable situation on the level of obtained mixed level in the case of further separation of the part and substrate can be Inverse Gaussian laser beam, which reduces the thickness of the mixed layers. For the case of growing monostructures (the same material for a part and a substrate) Flat-top laser beam mode can be preferable one.

The micro defects of the objects' structure for Gaussian, Flat-top and Inverse Gaussian laser beam mode are presented in Figure 7. The structure of the sample produced by Gaussian laser beam mode (Fig. 7a) has granular character. The picture was obtained on the border between upper and mixed layers. There is solid solution of iron and CoCrMo, while the granules of iron was washed out by solution of etching. The structure of the samples for Flat-top and Inverse Gaussian laser beam mode (Figs. 7b and 7c) looks like homogeneous with dissemination pores. In the both cases, there are some flash points of pores diffusion, related probably to change of microstructure between solid solution of mixed layers (iron $+\mathrm{CoCrMo}$ ) and upper layers (CoCrMo). The average diameter of the pores is less than $1 \mu \mathrm{m}$. The pictures shows the possibility to obtain homogeneous microstructure of the samples with the usage of the power 150-170 W, which can be interesting for industrial applications for the purpose to reduce the operating time of SLM production of the objects.

\section{Conclusions}

The aim to describe the structure defects for 3Dobjects obtained by selective laser melting on the developed experimental setup was achieved. The investigation shows the difference between obtained microstructures and the defects related to the mixing of the materials between substrate and power were revealed. The research definitely showed that the thickness of the mixed layers of solid solution (powder material CoCrMo and substrate material steel 20 (AISI C1020)) for the objects reduces with extension of effective diameter of laser beam spot, obtained for different laser beam mode. It shows that the usage of Gaussian laser beam mode with its knife-like formation of the molten pool can be an advantage for laser cutting operation, but it is not preferable for the operation related to direct solid growing conception such as selective laser melting and laser cladding. Moreover, the usage of alternative laser beam modes gives a possibility to produce the objects with the laser power, which does not give any appropriate result for Gaussian laser mode (with the value of the power more than $150 \mathrm{~W}$ ).
Acknowledgements. This work has been financed by the Ministry of Education and Science of the Russian Federation in the frame of governmental task in the field of scientific activities (task order No. 9.811.2014/K).

The work is carried out on the equipment of the Center of collective use of MSTU "STANKIN".

\section{References}

[1] I. Yadroitsev, P. Bertrand, G. Antonenkova, S. Grigoriev, I. Smurov, Use of track/layer morphology to develop functional parts by selective laser melting, J. Laser Appl. 5 (2013) 052003

[2] M. Merklein, R. Plettke, D. Junker, A. Schaub, B. Ahuja, Mechanical Testing of Additive Manufactured Metal Parts, Key Eng. Mater. 651-653 (2015) 713-718

[3] I. Yadroitsev, A. Gusarov, I. Yadroitsava, I. Smurov, Single track formation in selective laser melting of metal powders, J. Mater. Process. Technol. 210 (2010) 16241631

[4] S.N. Grigoriev, V.I. Teleshevskii, Measurement problems in technological shaping processes, Measurement Techniques 54 (2011) 744-749

[5] S.N. Grigor'ev, T.V. Tarasova, G.O. Gvozdeva, S. Nowotny, Micro-Laser Facing of Al - Si System Alloys, Metal. Sci. Heat Treatment 55 (2013) 242-246

[6] Y. Lu, S. Wu, Y. Gan, S. Zhang, S. Guo, J. Lin, J. Lin, Microstructure, mechanical property and metal release of As-SLM CoCrW alloy under different solution treatment conditions, J. Mech. Behavior Biomedical Mater. 55 (2016) 179-190

[7] Y. Chen, Y. Li, S. Kurosu, K.Yamanaka, N. Tang, Y. Koizumi, A. Chiba, Effects of sigma phase and carbide on the wear behavior of CoCrMo alloys in Hanks' solution, Wear 310 (2014) 51-62

[8] W.E. King, A.T. Anderson, R.M. Ferencz, N.E. Hodge, C. Kamath, S.A. Khairallah, A.M. Rubenchik, Laser powder bed fusion additive manufacturing of metals; physics, computational, and materials challenges, Appl. Phys. Rev. 2 (2015) 041304

[9] T.V. Tarasova, Prospects of the use of laser radiation for raising the wear resistance of corrosion-resistant steels, Metal Sci. Heat Treatment 52 (2010) 284-288

[10] C.Y. Han, Y. Ishii, K. Murata, Reshaping collimated laser beams with Gaussian profile to uniform profiles, Appl. Opt. 22 (1983) 3644-3647

[11] P.W. Rhodes, D.L. Shealy, Refractive optical systems for irradiance redistribution of collimated radiation: their design and analysis, Appl. Opt. 19 (1980) 3545-3553

[12] A. Hendriks, D. Naidoo, F.S. Roux, C. López-Mariscal, A. Forbes, The generation of flat-top beams by complex amplitude modulation with a phase-only spatial light modulator, Proc. SPIE 8490 (Laser Beam Shaping XIII) (2012) 849006

[13] T.V. Tarasova, E.V. Popova, Formation of Coatings by a Method of Overlay Laser Welding of Powders of Titanium and Silicon Carbide on a Surface of Low-Carbon Steel, Metallofizika I Noveishie Tekhnologii 35 (2013) 14871500

[14] R.G. Bohatch, K. Graf, A. Scheid, Effect of Track Overlap on the Microstructure and Properties of the CoCrMoSi PTA Coatings, Mat. Res. 18 (2015) 
[15] R. Voelkel, K.J. Weible, Laser Beam Homogenizing: Limitations and Constraints, Proc. SPIE 7102, Optical Fabrication, Testing, and Metrology III, 71020J (September 25, 2008)

[16] H. Ma, Z. Liu, P. Zhou, X. Wang, Y. Ma, X. Xu, Generation of flat-top beam with phase-only liquid crystal spatial light modulators, J. Opt. 12 (2010) 045704

[17] R. Bourouis, K. Ait Ameur, H. Ladjouze, Optimization of the Gaussian beam flattening using a phaseplate, J. Mod. Opt. 44 (1997) 1417-1427

[18] D.L. Shealy, J.A. Hoffnagle, Laser beam shaping profiles and propagation, Appl. Opt. 45 (2006) 5118-5131

[19] A. Okunkova, M. Volosova, P. Peretyagin, Yu. Vladimirov, I. Zhirnov, A.V. Gusarov, Experimental approbation of selective laser melting powders by usage of non-Gaussian power density distribution, Phys. Proc. 56 (2014) 48-57

[20] A. Okunkova, P. Peretyagin, Yu. Vladimirov, M. Volosova, R. Torrecillas, S.V. Fedorov, Laser-beam modulation to improve efficiency of selecting laser melting for metal powders, Proc. SPIE 9135 Laser Sources and Applications II (May 1, 2014) 913524
[21] I.V. Zhirnov, P.A. Podrabinnik, A.A. Okunkova, A.V. Gusarov, Laser beam profiling: experimental study of its influence on single-track formation by selective laser melting, Mechanics \& Industry 16 (2015) 709

[22] P. Yu. Peretyagin, I.V. Zhirnov, Yu. G. Vladimirov, T.V. Tarasova, A.A. Okun'kova, Track geometry in selective laser melting, Russian Eng. Res. 35 (2015) 473-476

[23] A.V. Gusarov, A.A. Okun'kova, P.Yu. Peretyagin, I.V Zhirnov, P.A. Podrabinnik, Means of Optical Diagnostics of Selective Laser Melting with Non-Gaussian Beams, Measurement Techniques 58 (2015) 872-877

[24] I.V. Zhirnov, P.A. Podrabinnik, M. Tokbergenov, A.A. Okunkova, I.Y. Smurov, Optical Monitoring and Diagnostics of SLM Processing for Single Track Formation from Co-Cr Alloy, Mater. Sci. Forum 834 (2015) 51-60

[25] A. Okunkova, M. Volosova, P. Peretyagin, I. Zhirnov, P. Podrabinnik, S.V. Fedorov, A. Gusarov, Study of laser beam modulation influence on structure of materials produced by Additive Manufacturing, Adv. Mater. Lett. 7 (2016) 111-115 